\title{
Esophageal Actinomycosis: A Case Report
}

Actinomycosis usually causes infection in the face, lung, and abdomen. Esophageal actinomycosis is rare, and only three cases have been reported in the past 20 years $(1,2)$. All were patients with AIDS. We report here the case of a 55-year-old man with mid-esophageal infection by Actinomyces viscosus.

The patient presented with epigastric pain and weight loss that had persisted for three months. The physical examination and basic tests, including blood count, renal and liver biochemistry, and chest radiography, were entirely normal. Upper endoscopy revealed a $1-\mathrm{cm}$ erythematous nodule situated at the mid-esophagus (Figure 1). Biopsy showed a clumped bacterial colony overlying an inflamed esophageal squamous epithelium. Culture confirmed the presence of Actinomyces viscosus (Figure 2). Endoscopic ultrasonography demonstrated that the nodule was a $1-\mathrm{cm}$ cavitating lesion, arising from the third layer of the esophageal wall (Figure 3). Abdominal ultrasonography and a CT of the thorax were unremarkable. The patient declined an HIV test. He was treated with oral co-amoxiclav (amoxycillin and clavulanic acid) $375 \mathrm{mg}$ thrice daily. After four weeks of treatment, a repeat upper endoscopy confirmed complete resolution of the lesion. A further biopsy was negative for Actinomyces. Three months later, he developed painless obstructive jaundice. Disseminated pancreatic adenocarcinoma was diagnosed at laparotomy, and he died one month later.

Actinomyces infection occurs in both immunocompetent and immunocompromised hosts (3). Impairment of cytotoxic T-cell function, as reported in patients with pancreatic carcinoma (4), may have been the predisposing factor in our patient. As the esophageal nodule was situated at one of the three common sites of foreign-body impaction, we postulate that a minor mucosal injury resulting from normal deglutition had allowed entrance of the Actinomyces organism (5). A search for an underlying malignancy may be rewarding if actinomycosis is found at an unusual site.

\section{F. H. Ng', S. Y. Wong', C. M. Chang', S. T. Lai ${ }^{2}$, K. Y. Chau ${ }^{3}$ ' Dept. of Medicine, Ruttonjee Hospital, Hong Kong \\ ${ }^{2}$ Dept. of Medicine, Princess Margaret Hospital, Hong Kong \\ ${ }^{3}$ Dept. of Pathology, Queen Mary Hospital, Hong Kong}

\section{References}

1. Poles MA, McMeeking AA, Scholes JV, Dieterich DT Actinomyces infection of a cytomegalovirus esophageal ulcer in two patients with acquired immunodeficiency syndrome. Am J Gastroenterol 1994; 89: 1569-72.

2. Spencer GM, Roach D, Skucas J. Actinomycosis of the esophagus in a patient with AIDS: findings on barium esophagogram. Am J Roentgenol 1993; 16: 795-6.

3. Weese WC, Smith IM. A study of 57 cases of actinomycosis over a 36-year period: A diagnostic "failure" with good prognosis after treatment. Arch Intern Med 1975; 135: 1562

4. Tempero MA, Sivinski C, Steplewski Z, Harvey E, Klassen L, Kay HD. Phase II trial of interferon gamma and monoclonal antibody $17-1 \mathrm{~A}$ in pancreatic cancer: biologic and clinical effect. J Clin Oncol 1990; 8:2019-26.

5. Palmer ED. Actinomycosis. In: The esophagus and its diseases. New York: Hoeber, 1952: 281-2

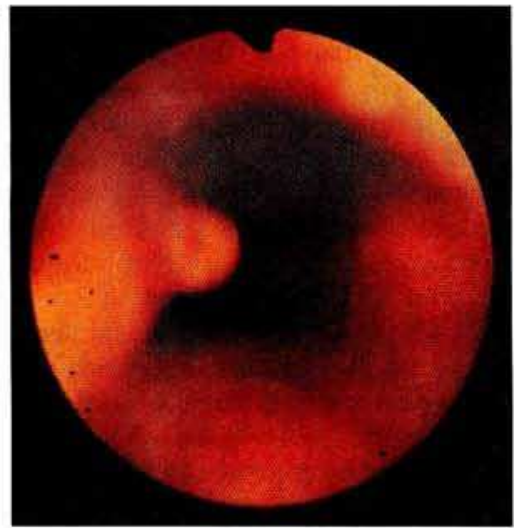

Figure 1: Endoscopic image of the esophageal nodule situated in the midesophagus

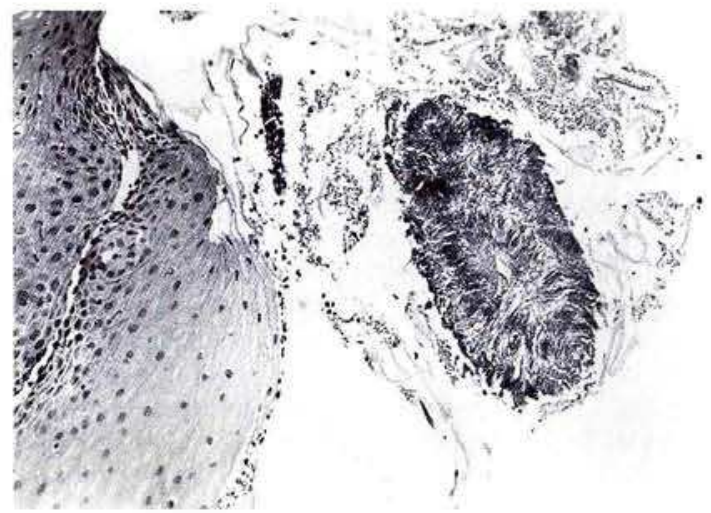

Figure 2: Microscopically, a clumped bacterial colony with the characteristics of Actinomyces species overlay an inflamed esophageal squamous epithelium.

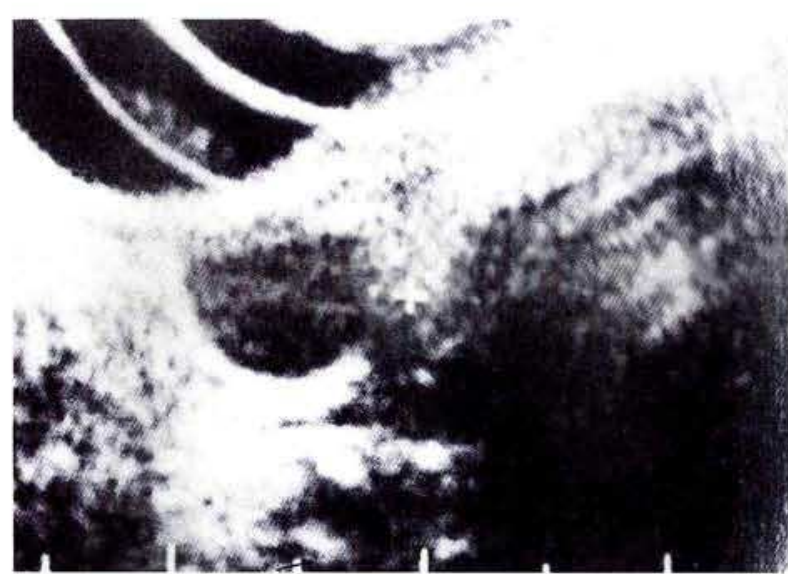

Figure 3: A $1 \mathrm{~cm}$ cavitating lesion, arising at the third layer of the esophageal wall.

Corresponding Author

F. H. Ng, M.B., B.S., M.R.C.P.

Dept. of Medicine, Ruttonjee Hospital

226 Queen's Road East, Hong Kong

Fax: +852-2836-1363 